Sir — We should like to correct a statement in your Briefing on malaria about funding for the sequencing of the genome of the malaria parasite Plasmodium falciparum (Nature 386, 535-540; 1997).

You report that the US Department of Defense, the US Burroughs Wellcome Fund, the UK Wellcome Trust and the US National Institutes of Health (NIH) have created a fund for this sequencing effort. In fact, no 'fund' has been established and there has been no pooling of funds among the donors. However, an exciting international consortium of scientists and funding agencies has been formed during the past year to provide the funds and reagents required to sequence the $P$. falciparum genome, and to produce, annotate and publish these sequences.

We estimate that it will cost a minimum of $\$ 15$ million to complete and annotate the 30-megabase P. falciparum genome. In the initial pilot phase, funding has been used to develop methods and to produce the reagents required for the high-throughput sequencing and for annotating the sequences. As a result of progress so far, the plan is to sequence the 14 chromosomes separately and to divide the work by chromosome, with about half the work being done in the United States and half in England. The work in the United States has initially been supported by grants from the $\mathrm{NIH}$ and the Burroughs Wellcome Fund and in England by the Wellcome Trust. Subsequent support will also be provided by the US Department of Defense. In the United States, the Naval Medical Research Institute is providing $P$. falciparum DNA and chromosomes, the Institute for Genomic Research will be doing a significant amount of the high-throughput sequencing and Stanford University is committed to sequencing at least one of the larger chromosomes.

The donors are also supporting research on clone stability, library construction and optimization of sequencing reagents for the project at these three institutions, and at Roswell Park Cancer Institute and Harvard University. In England, the Institute of Molecular Medicine at the University of Oxford is providing $P$. falciparum reagents, and sequencing is being done at the Sanger Centre at Cambridge. It is expected that during the next few years a number of other laboratories may participate in the effort.

The participants (scientists and donors) involved in this collaborative undertaking coordinate their activities through conference calls, e-mails and meetings. The researchers and funders met in Baltimore, Maryland, in December 1996 and will meet in Cambridge, England, on 16-17 June 1997. In addition, there has been an effort to involve the broader malaria community in this project and to facilitate widespread dissemination of the genomic information.

There are substantial scientific obstacles to be overcome before the sequence of the $P$. falciparum genome is fully elucidated. Nonetheless, during the past year we have made great strides in identifying the funds required to pursue the project, building a strong group of scientists and institutions to execute the science, and establishing a collaborative network of scientists and donors. We believe that this project will provide a road map for malaria research in the twentyfirst century, research that will lead to improved treatment and prevention of a parasitic infection that causes hundreds of millions of illnesses, and millions of deaths annually.

Stephen L. Hoffman

Malaria Program,

Naval Medical Research Institute,

Bethesda,

Maryland 20889-5607, USA

e-mail:hoffmans@nmripo.nmri.nnmc.navy.mil William H. Bancroft

Military Infectious Disease Research Program, US Army Medical Research and

Materiel Command,

Frederick, Maryland 21703, USA

Michael Gottlieb

Stephanie L. James

Parasitology and International Programs Branch, NIAID/NIH,

Bethesda, Maryland 20892, USA

Enriqueta C. Bond Burroughs

Wellcome Fund,

Morrisville, North Carolina 27560, USA

John R. Stephenson

Michael J. Morgan

The Wellcome Trust,

London NW1 2BE, UK

\section{NERC maligned}

Sir - Tina Yates' statement (Nature 386, 754 ; 1997) that directors within the Natural Environment Research Council (NERC) are not concerned to create "an environment and career structure within which scientists can flourish over the longer term" is wrong.

In the NERC Centre for Coastal and Marine Sciences (CCMS), we have recently offered open-ended employment to 24 out of 36 ( 66 per cent) fixed-term (that is, impermanent) staff of more than five years' standing on contract. Just four (11 per cent) of the contracts have been allowed to expire; the remainder have been renewed for later review. This action is consistent with both the spirit and the letter of NERC policy.

Further, at a time of severe pressure on our core budget, it amounts to an expression of confidence by institute directors in the role of young scientists in maintaining the scientific vigour of the laboratories. Young research staff employed on contract have never been viewed by the CCMS laboratories as some sort of flexibility buffer. On the contrary, they are an essential part of the research life of any active laboratory, and we have welcomed the opportunity to give practical expression to this view through the recent NERC employment policy.

Brian L. Bayne

(Director)

Centre for Coastal and Marine Sciences,

Prospect Place, West Hoe,

Plymouth PL1 3DH, UK

\section{Once upon a time...}

Sir - None of the discussions I have seen about the US government's plans to spend $\$ 4$ billion a year maintaining the safety and reliability of its stockpile of nuclear weapons has pointed out that once upon a time a small group of scientists with no prior experience in the field and using 1940s knowledge and technology and no computers at all managed in the space of about three years to design two completely different atomic bombs, both of which worked spectacularly on the first try.

How can it now require a huge experienced design staff and new computers and experimental devices worth billions to assure the continued availability of weapons already designed and well tested? Such a claim certainly does not seem to attribute very much competence to those in the field today.

The only people who need $\$ 4$ billion a year spent on stockpile stewardship are those in whose pockets those dollars will end up and the politicians who want their votes.

\section{Bill Mixon}

14045 North Green Hills Loop,

Austin, Texas 78737, USA

e.mail:billmixon@worldnet.att.net 